\section{Background and Objective}

Metformin is widely used as oral anti-hyperglycaemic agent to treat Type 2 diabetes, with increasing reports of an additional, potential bone protective role. On a cellular level, mesenchymal stem cells (MSCs) have been reported to have reciprocal differentiation into bone at the expense of fat, and vice versa. We set out to investigate the effects of metformin on the multipotent mesenchymal stem cell differentiation and the underlying molecular mechanism(s) involved.

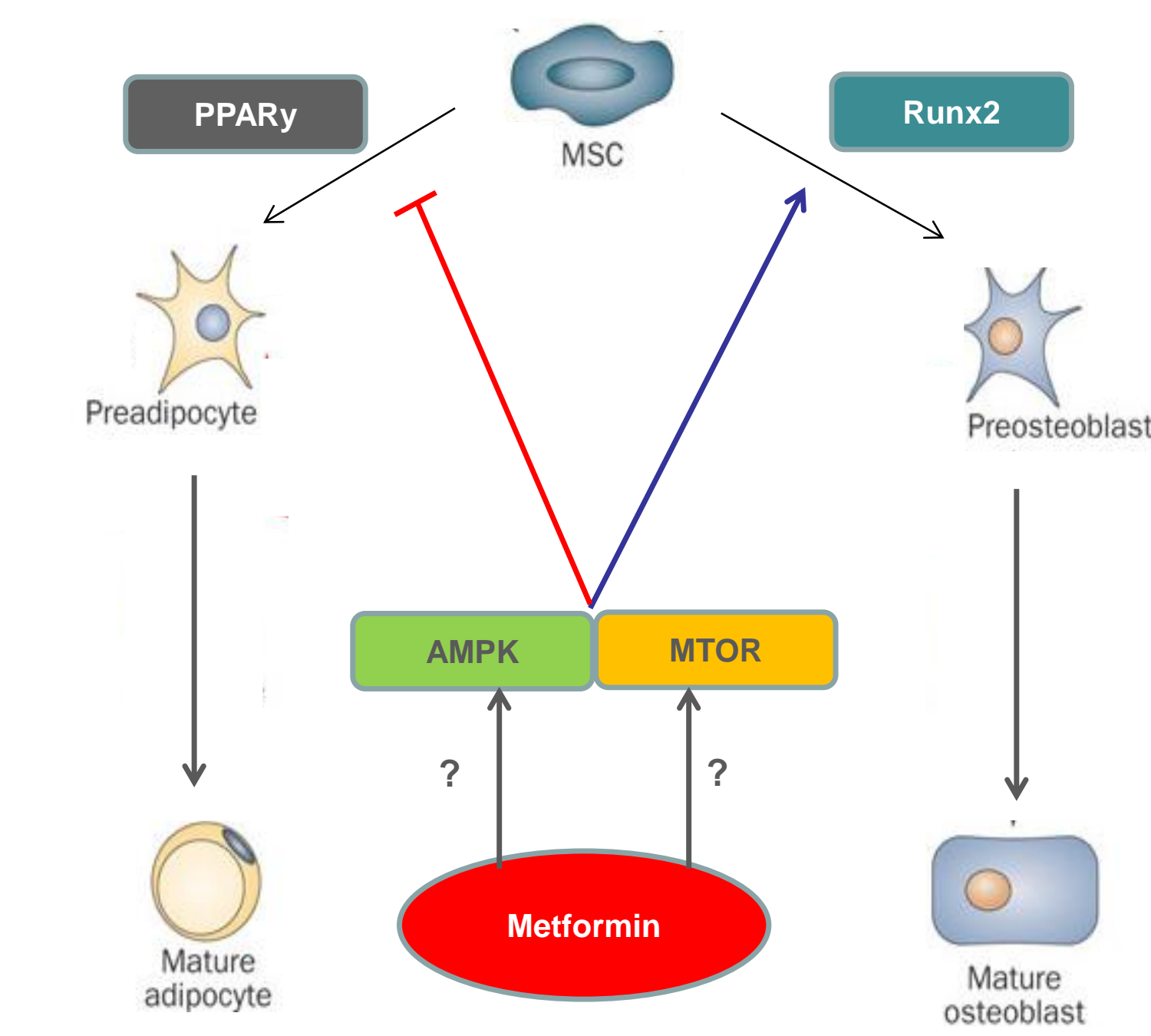

\section{Methods}

Confluent murine MSCs (C3H10T1/2) were treated with metformin $(500 \mu \mathrm{M})$, a known AMPK activator $(\mathrm{A} 769662 ; 100 \mu \mathrm{M})$ or the p70S6K inhibitor (rapamycin;10 $\mu \mathrm{M}$ ), in both control and adipogenic-inducing environments (using pioglitazone;10 $\mu \mathrm{M}$ ) for 5 days. Nuclear extracts were separated by SDS-PAGE and immunoblotted with primary antibodies to peroxisome proliferator-activated receptor gamma (PPARy;marker for adipogenesis), Runt-related transcription factor 2 (Runx2;marker for osteogenesis), phosphorylated-ACC (P-ACC(Ser79);marker for AMPK activity) and phosphorylated-p70s6k (P-p70s6k(Thr389); upstream regulator of mTOR signalling). Immunoblots were scanned using a Licor fluorescent reader. PPARy and Runx2 activities were determined using Luciferase reporter assays and adipogenesis was quantified histochemically by staining neutral lipids with Oil Red $\mathrm{O}$.

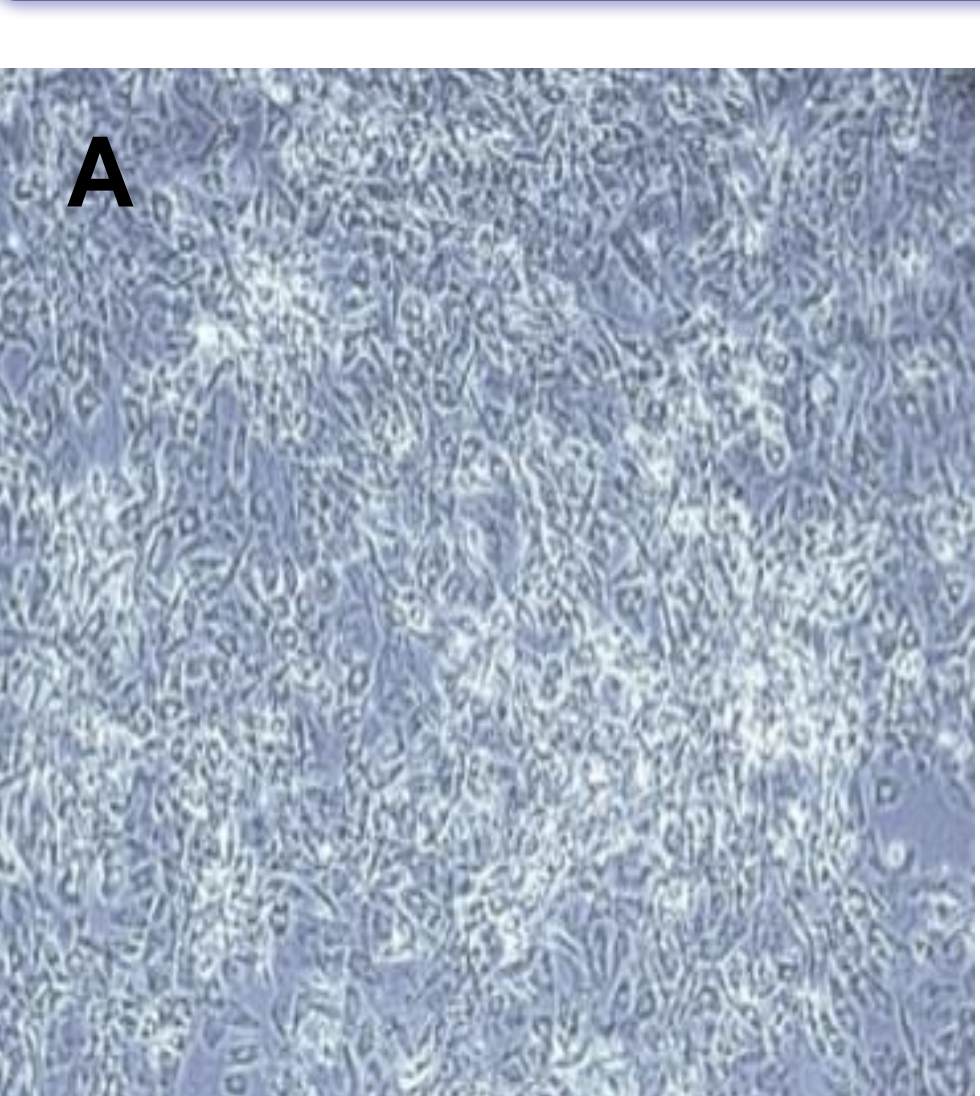

No Treatment

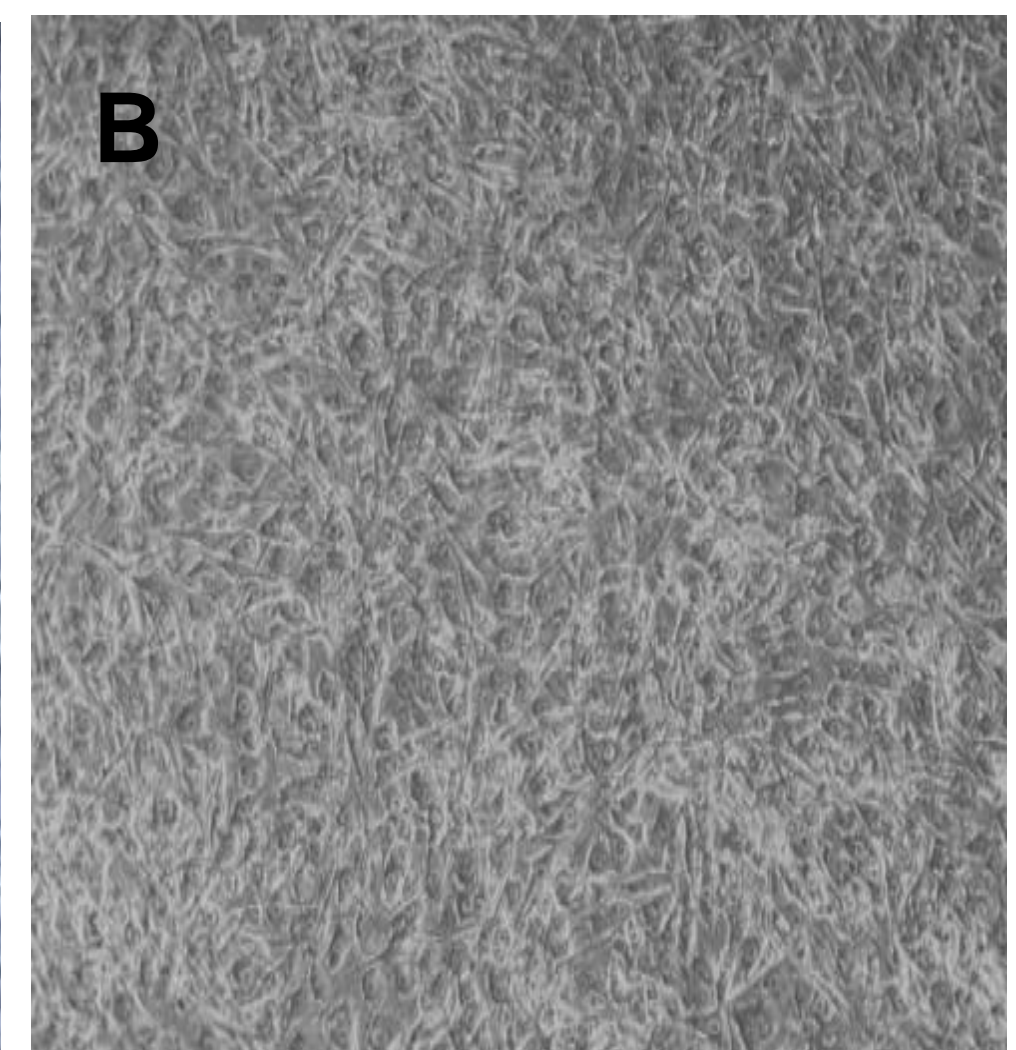

Metformin

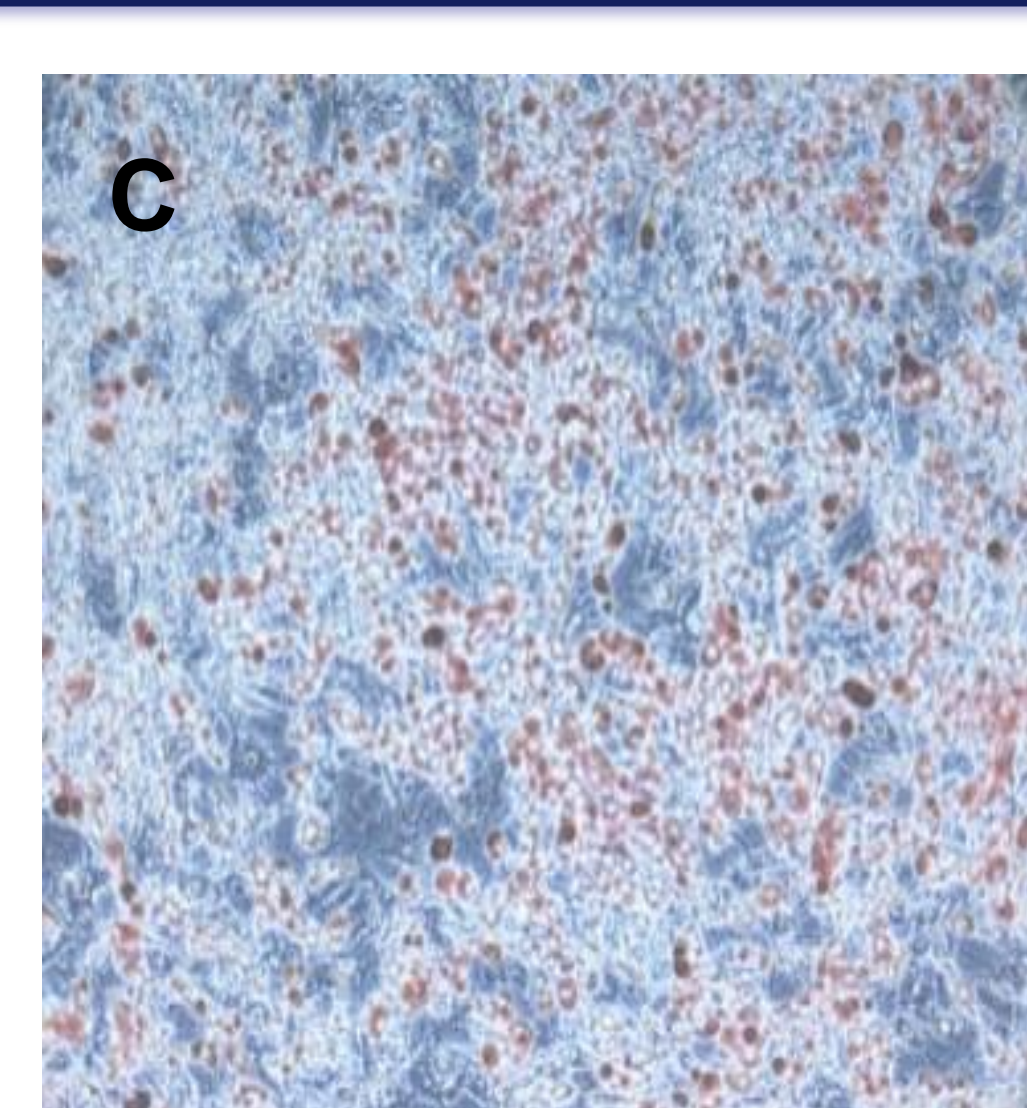

Pioglitazone

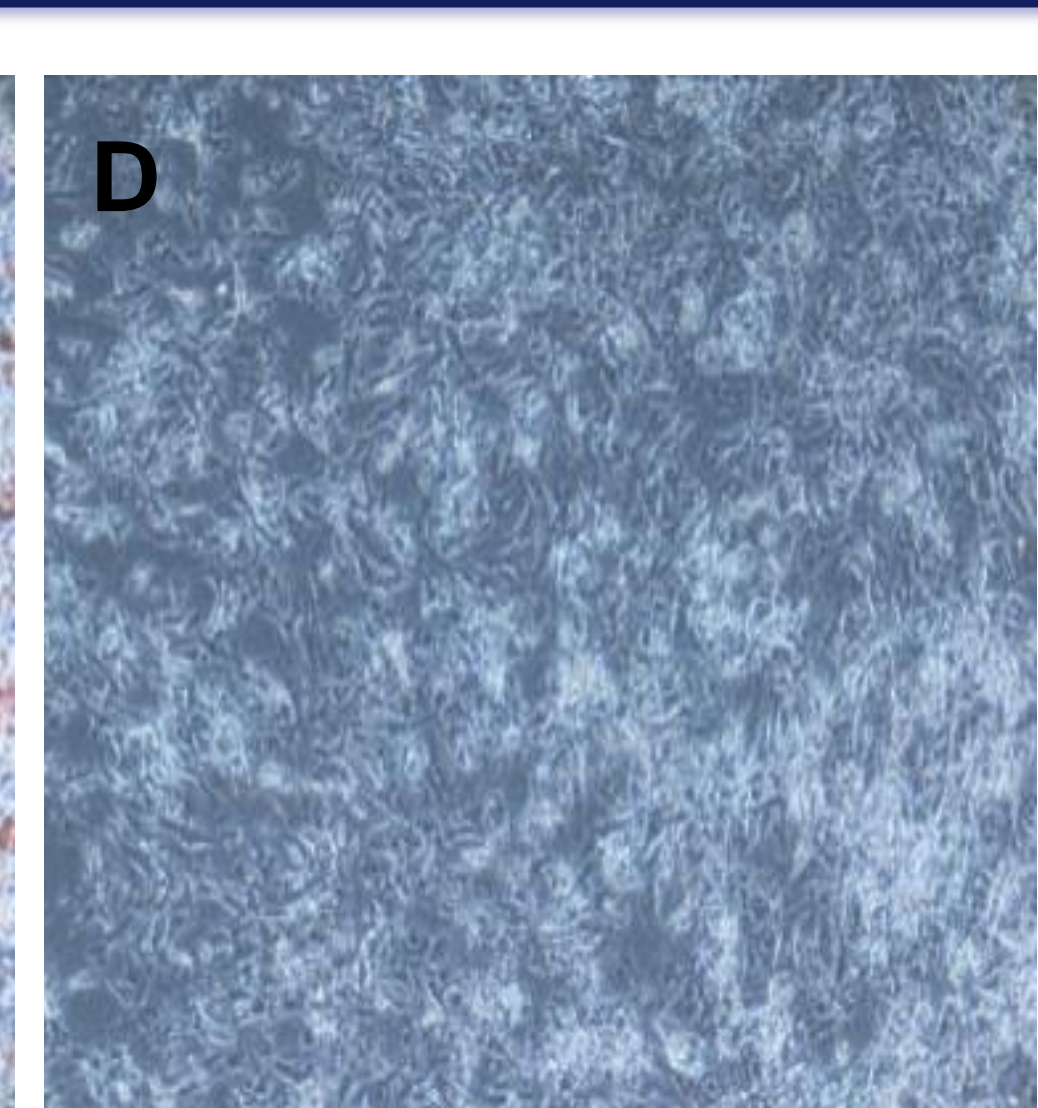

Pio + A769662

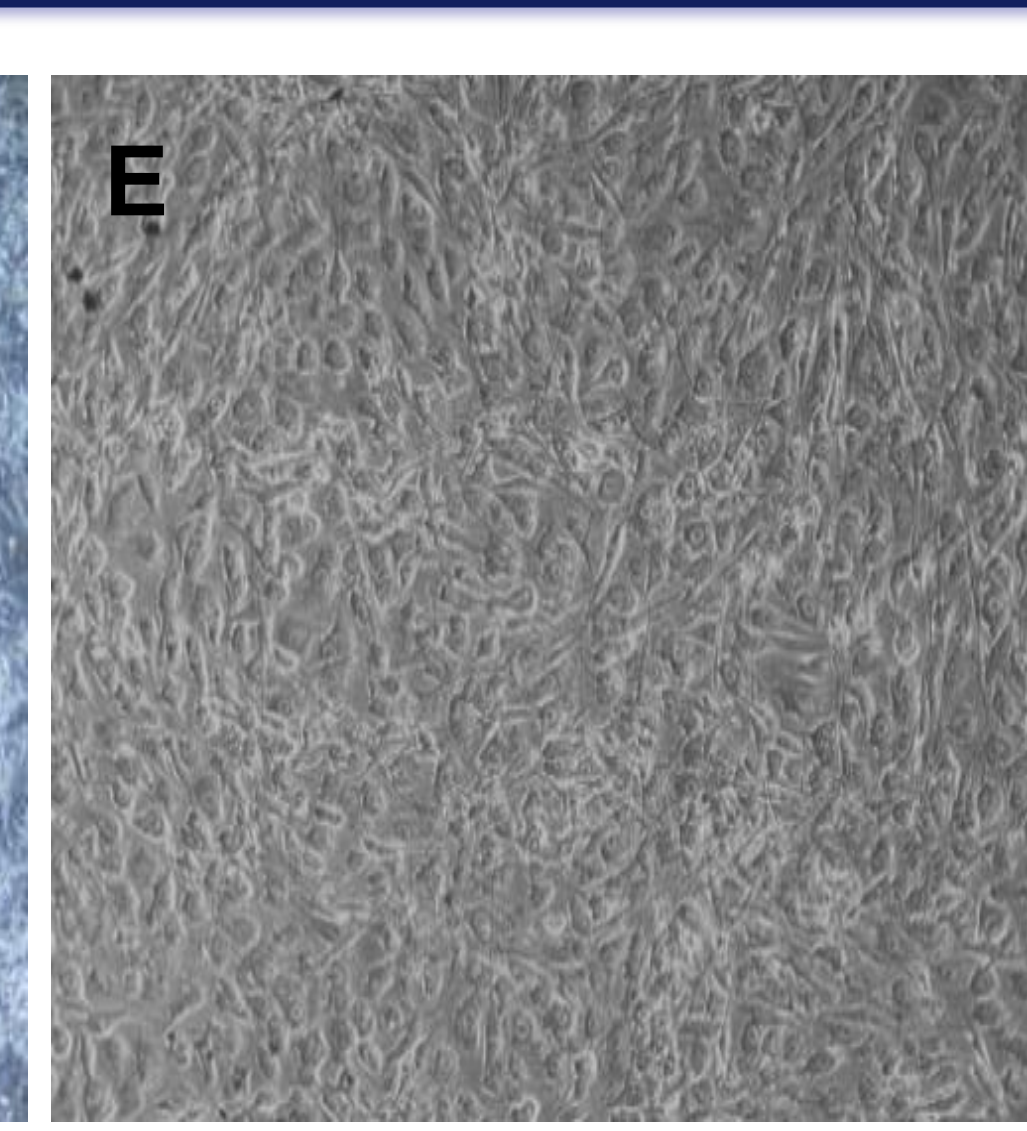

Pio + Metformin

Figure 1. Oil red $O$ staining on confluent C3H10T1/2 without treatment (A), metformin only (B), pioglitazone only (C), pioglitazone and AMPK activator A769662 (D), and pioglitazone and metformin (E). Pioglitazone exerts marked adipogenic effect whilst metformin and A769962 inhibits adipogenesis.

A $\operatorname{PPAR}$

Runx2

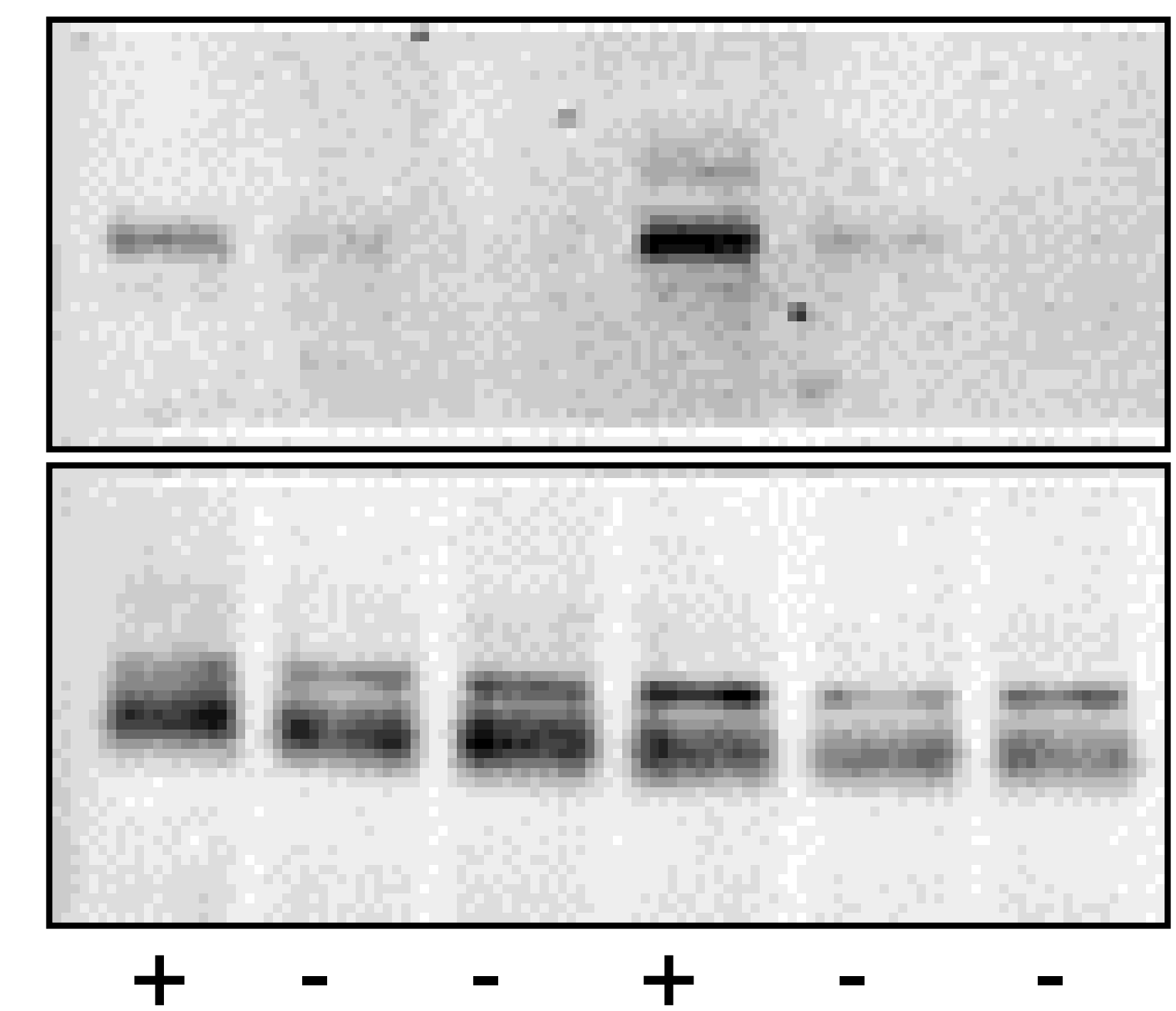

Metformin

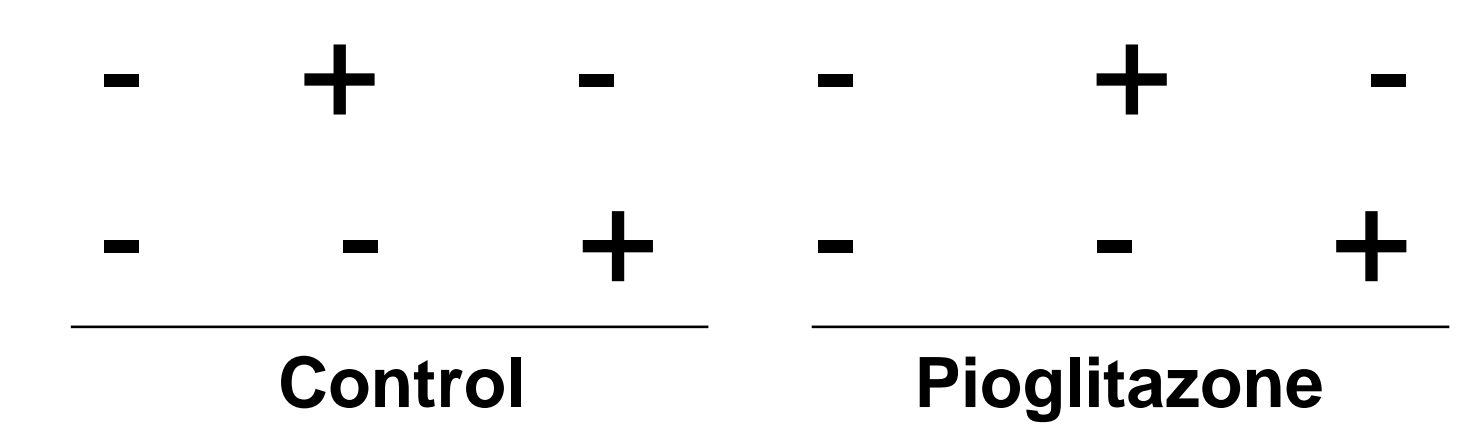

Figure 2. Protein expression of PPARy (marker of adipogenesis) and Runx2 (marker of osteogenesis) on Western blots (A) and quantification graphs. Metformin inhibits PPARy expression $(p<0.05)$, to a lesser degree compared to A76 $(p<0.01)(B 1)$, with no effect on Runx2 expression (B2).

PPARy

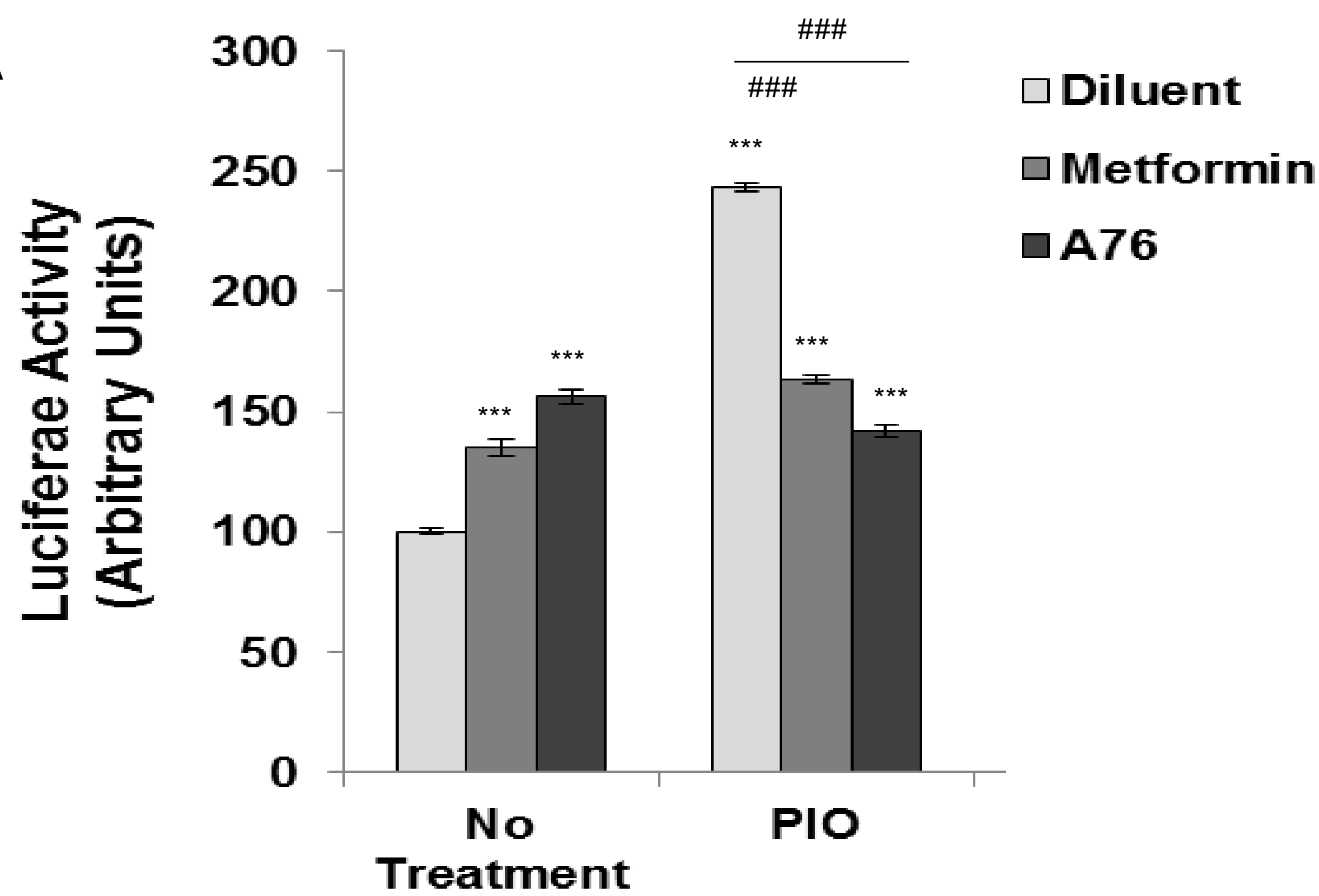

B1

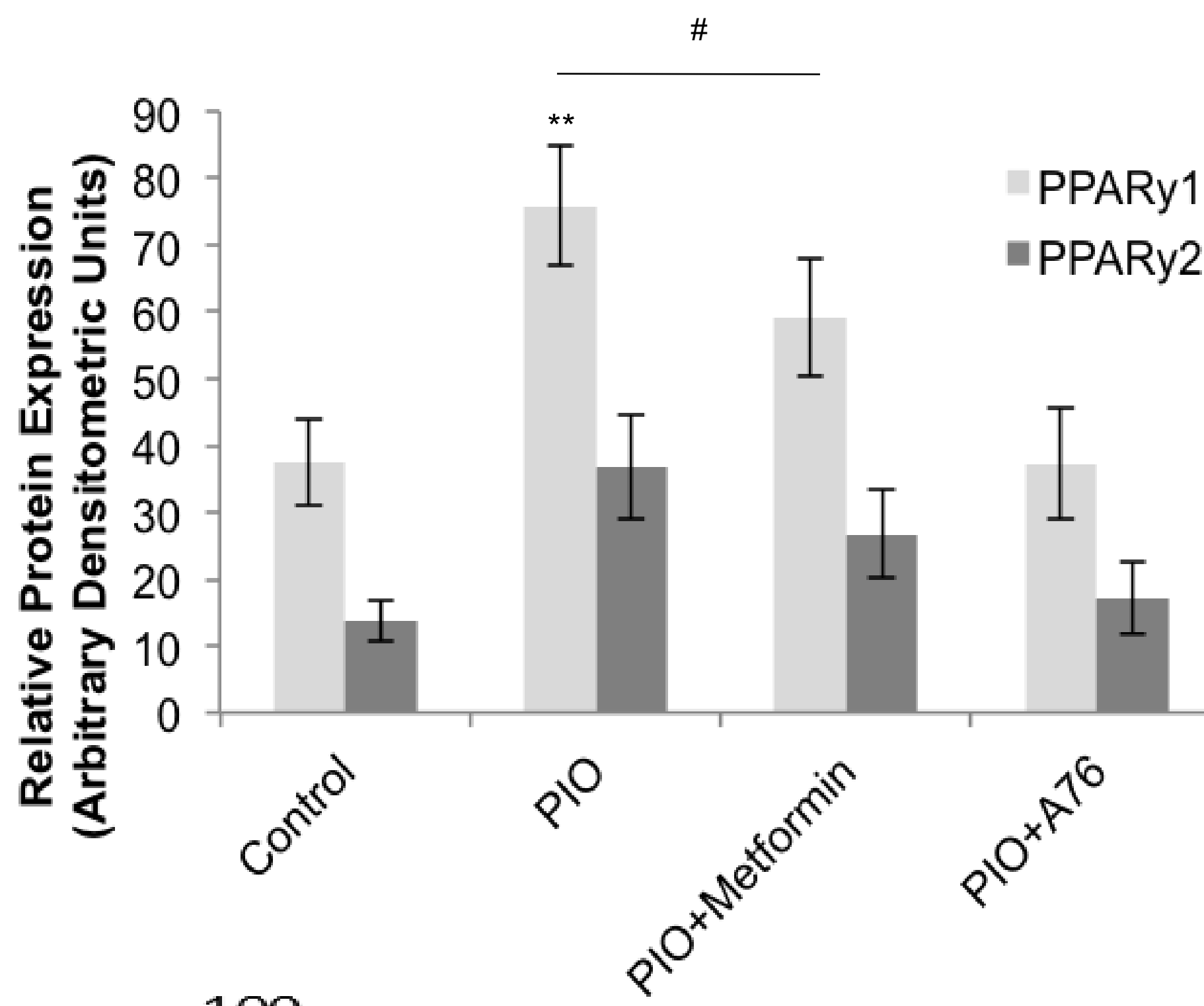

B2

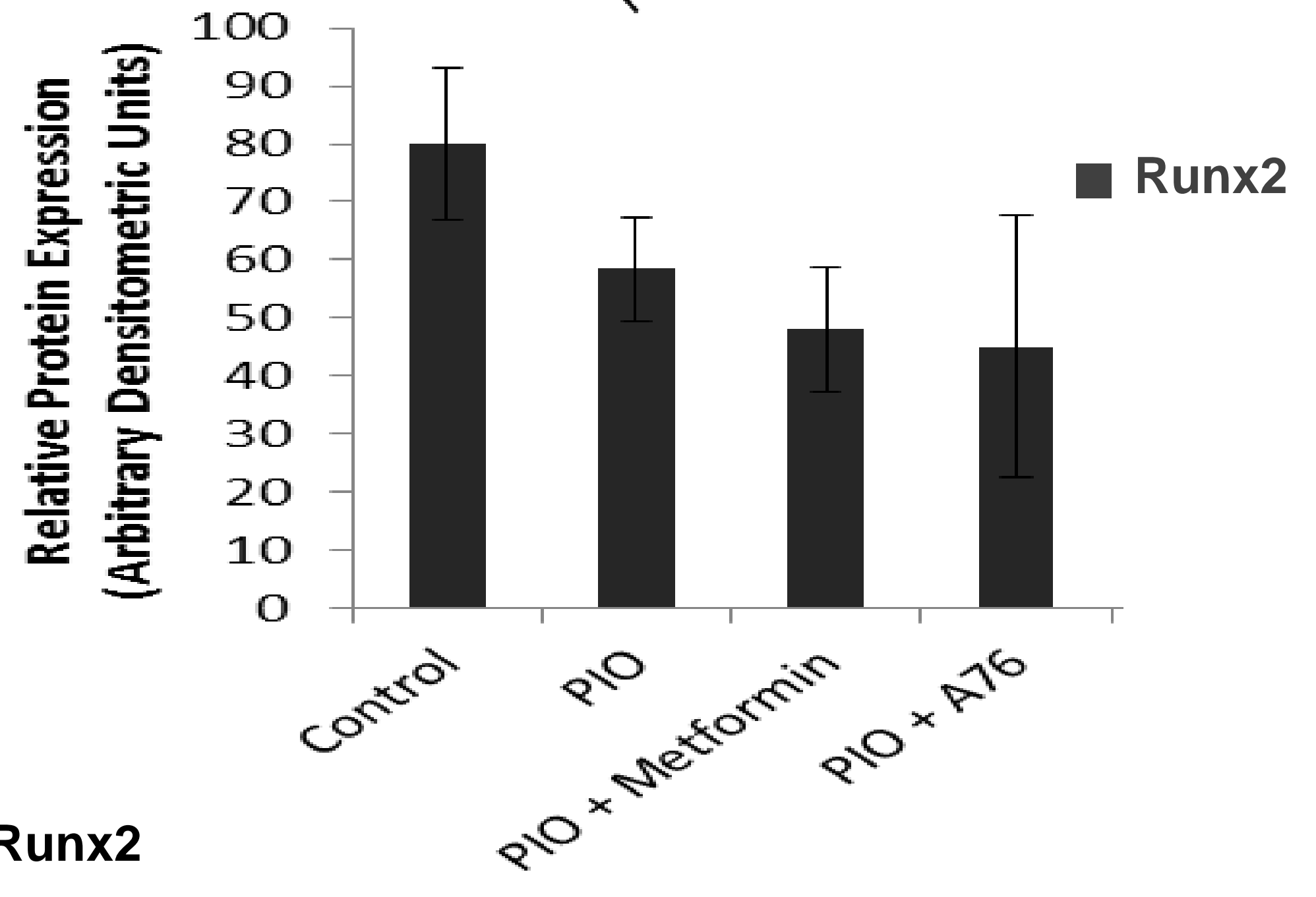

B

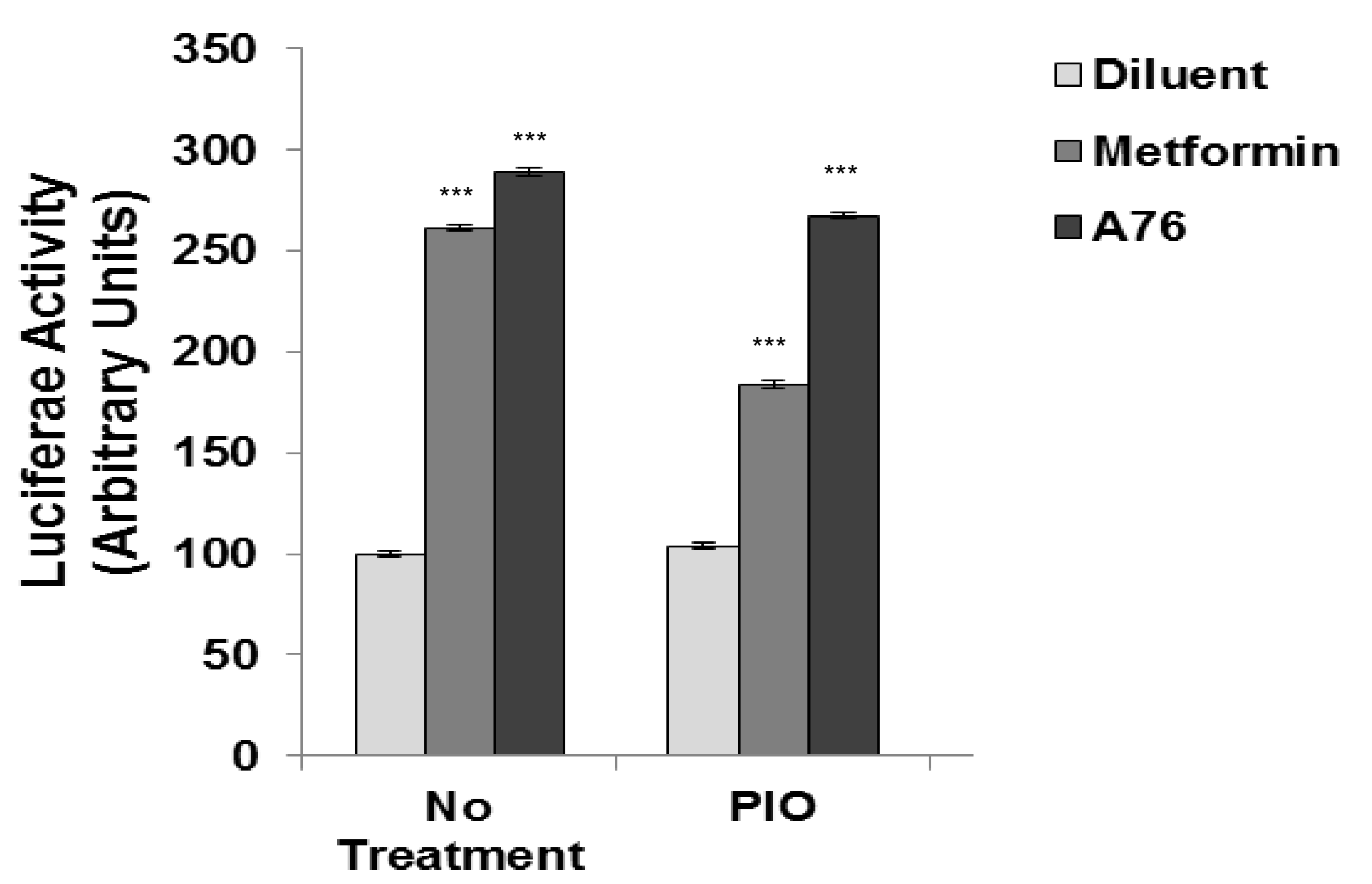

Figure 3. Luciferase assay to determine PPARy (marker of adipogenesis) and Runx2 (marker of osteogenesis) activities. Metformin reciprocally inhibits PPARy activity $(p<0.001)(A)$ and increases Runx2 activity $(p<0.001)(B)$. PACC TACC

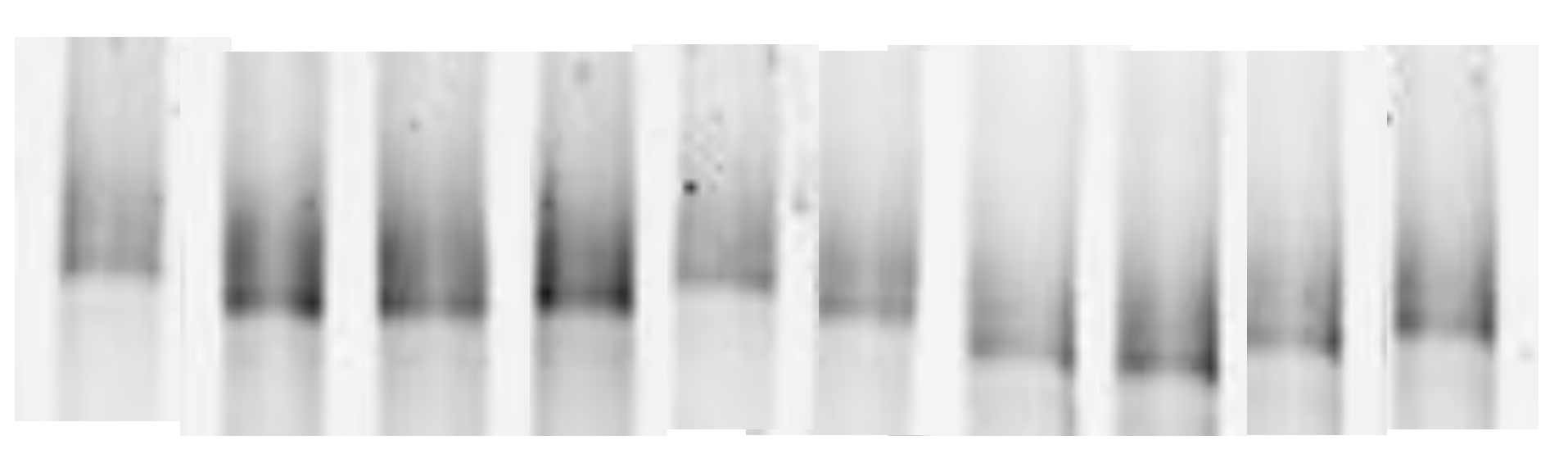

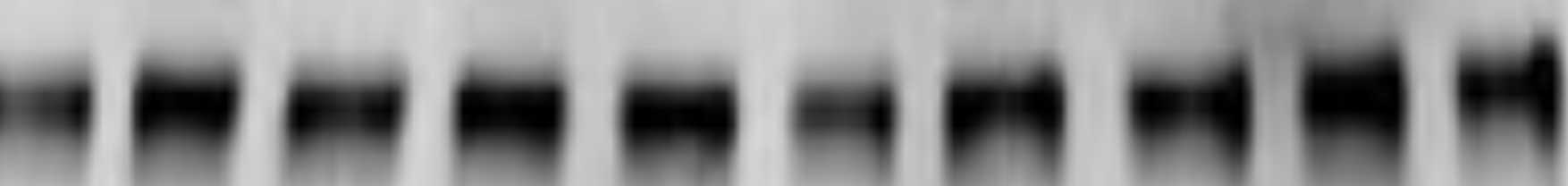

B

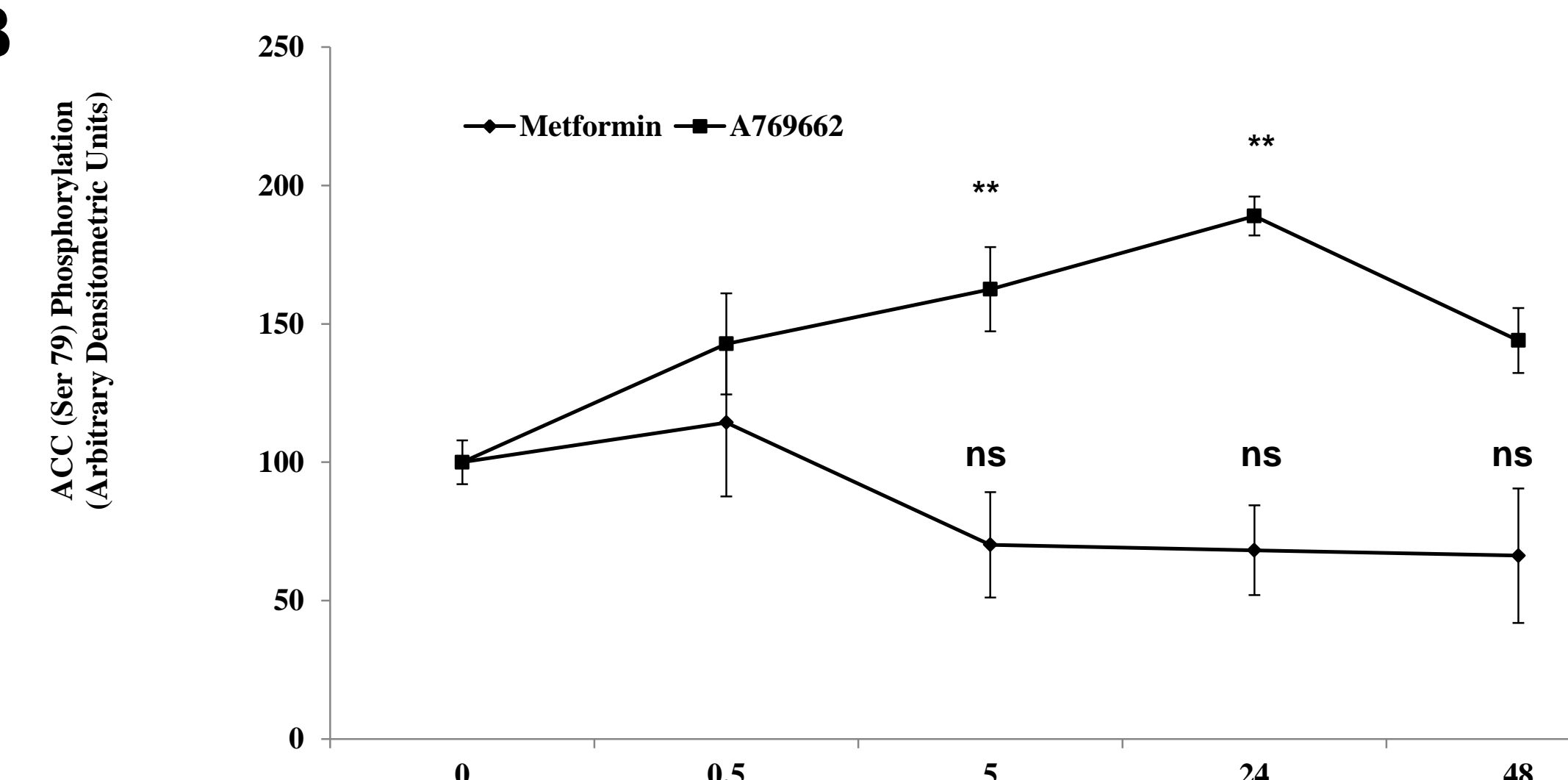

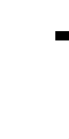

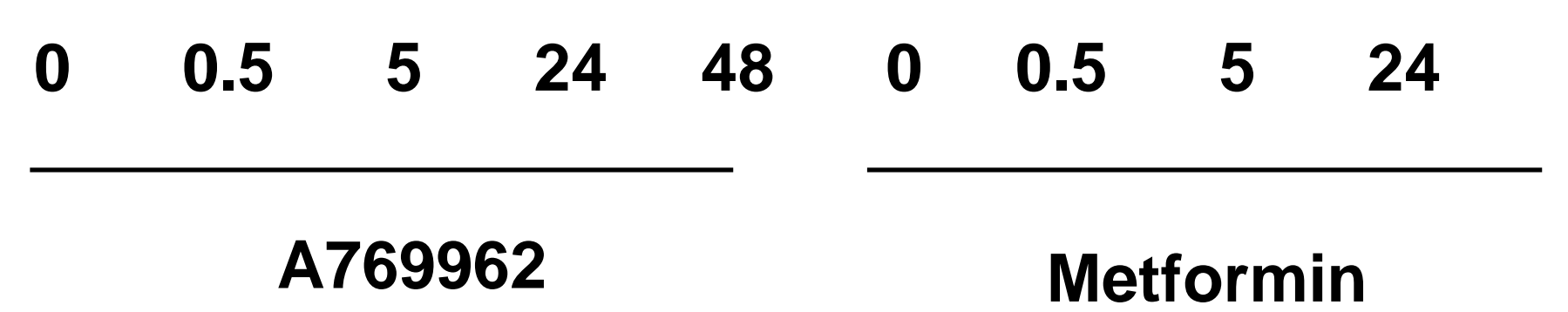

Figure 4. PACC expression (marker of AMPK activity) on Western blots (A) and quantification graphs. A769662 significantly increases expression of PACC, as expected, but only marginally so with metformin (B).
MSCs treated with pioglitazone demonstrated marked adipogenic phenotype staining positively with Oil Red $O$. In contrast, treatment with both metformin and A769662 impaired adipogenesis (Figure 1). Pioglitazone induced an $(p<0.01)$ increase in PPARy expression, whilst metformin and A796662 suppressed PPARy expression to basal levels, $p<0.05$ and $p<0.01$ respectively. Runx2 activity was significantly increased by metformin $(p<0.001)$ and $A 769662 \quad(P<0.001)$ but not Runx2 protein levels (Figure 2 and 3). As expected, A769662 promotes phosphorylation of ACC, but not so with metformin (Figure 4). Instead, metformin suppressed $(p<0.05)$ the phosphorylation of p70s6k, as did A769662 $(p<0.05)$ and rapamycin $(p<0.001)$ (Figure 6). Luciferase reporter assays confirmed the reciprocal action of metformin on adipogenesis and osteogenesis, namely suppression of PPARY activity $(p<0.001)$ and induction of Runx2 activity $(p<0.001)$.

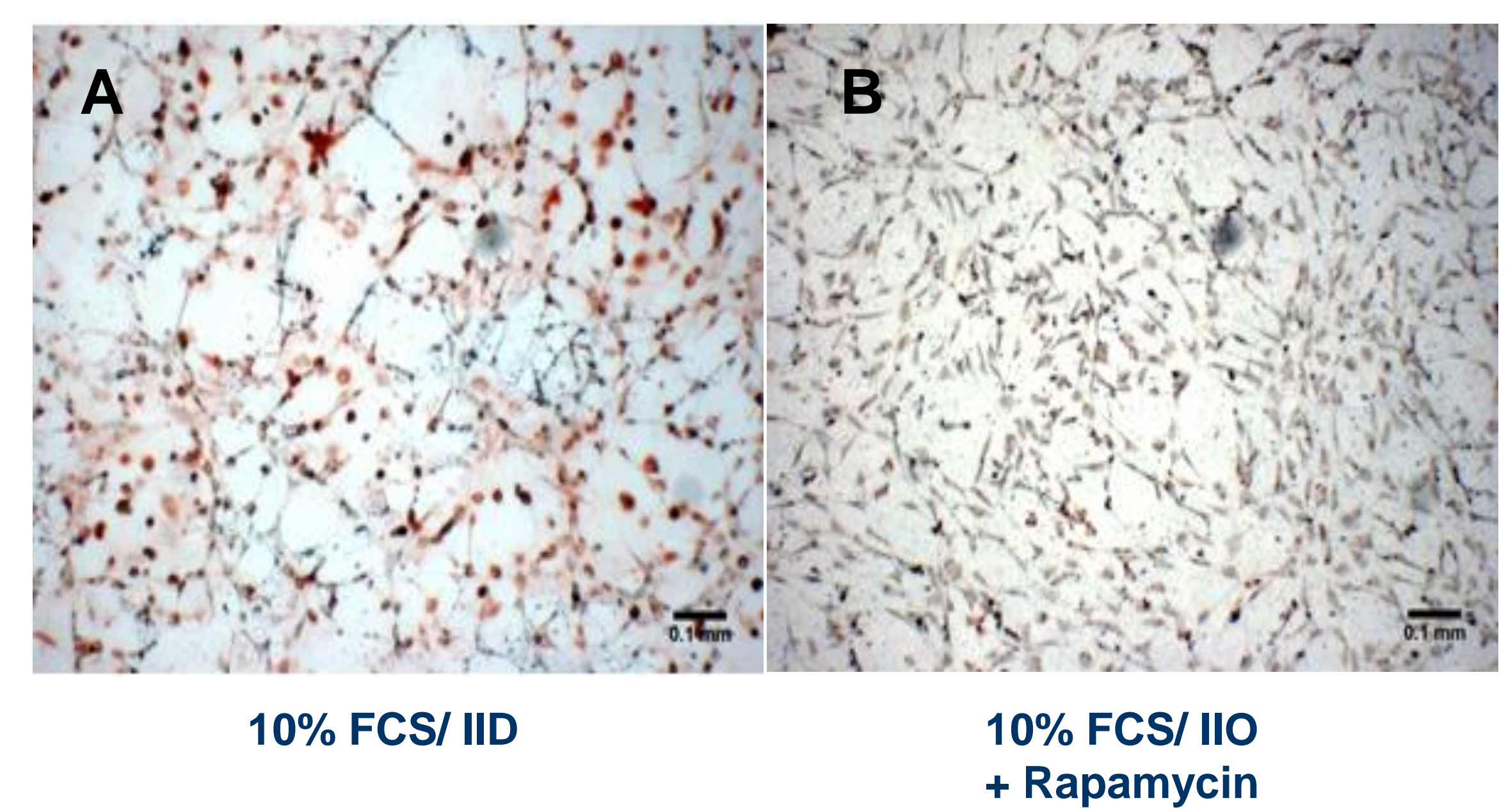

Figure 5. Oil red $O$ staining on confluent $\mathrm{C}_{3 \mathrm{H}} 10 \mathrm{~T} 1 / 2$ without treatment (A), and with rapamycin (B), Rapamycin, like metformin inhibits adipogenesis.

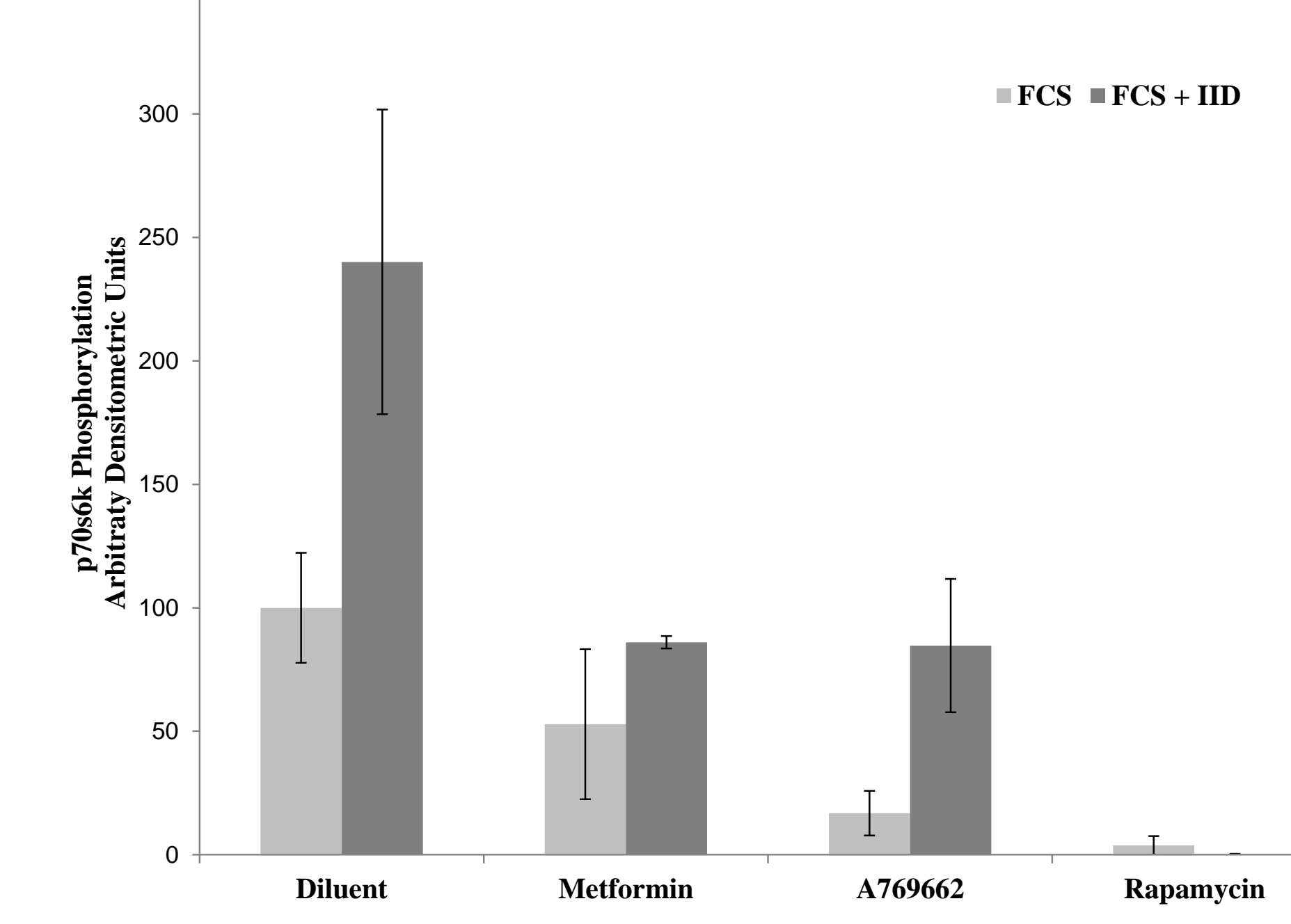

Figure 6. Metformin, like rapamycin, suppressed the phosphorylation of p70s6-kinase

\section{Conclusion}

- Metformin inhibits adipogenesis and promotes osteogenesis

- Acting through AMPK-independent pathway, involving the suppression of p70s6k in the mTOR signalling (novel mechanism of action)

- Rationale for the reported bone protective role of metformin

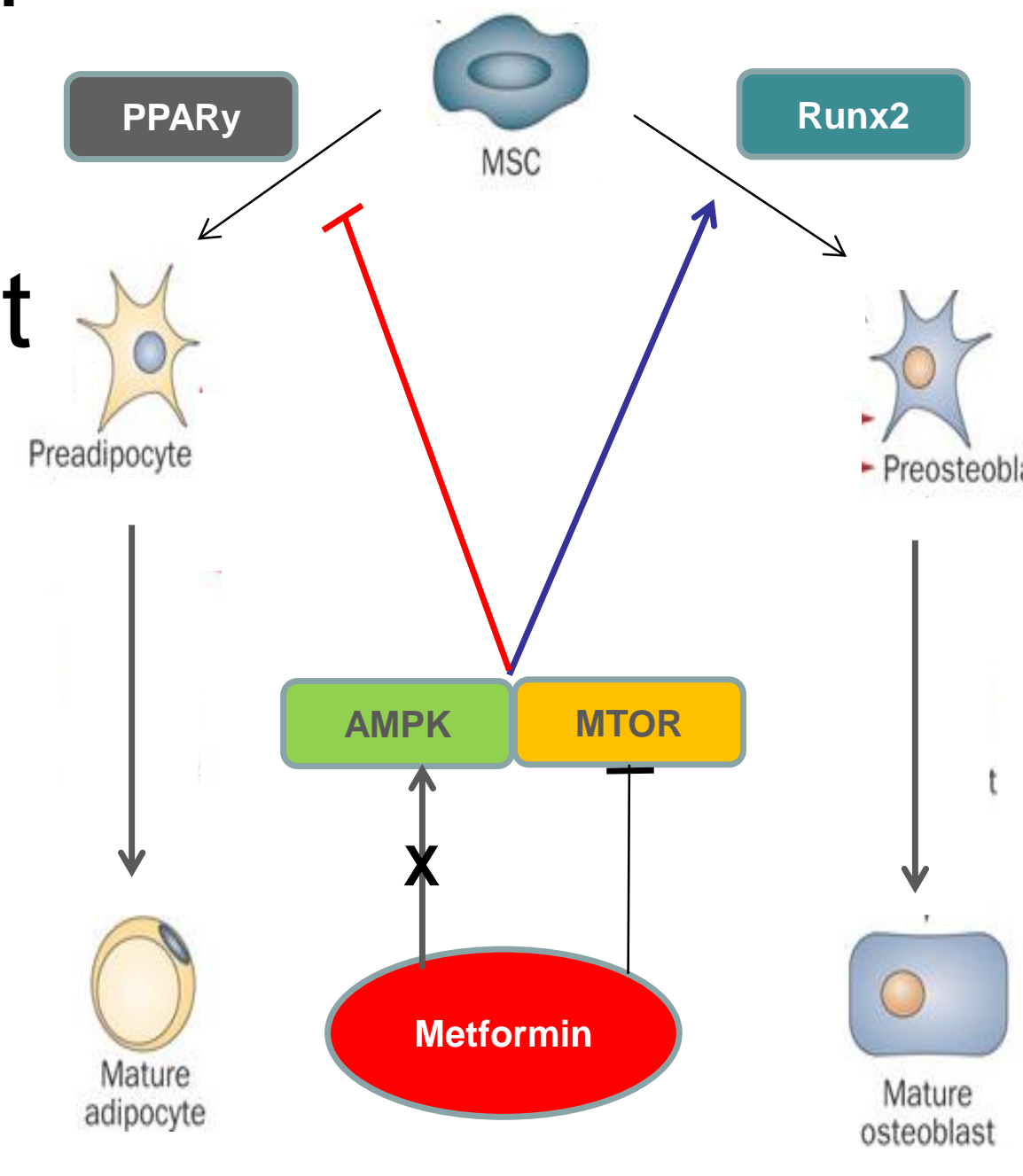

